\title{
Analysis of influencing factors on the efficacy of functional dyspepsia
}

\author{
Min Feng \\ Shandong Zibo City Center Hospital Digestion in a Family, Zibo, \\ Shandong, 255036
}

\begin{abstract}
A At present, the treatment of functional dyspepsia (FD) is not satisfactory, and efficacy-related factors of less research, in order to alleviate symptoms and improve quality of life, this study tries to evaluate the efficacy of functional dyspepsia related effects factor.
\end{abstract}

Keywords: Functional Dyspepsia, Influencing Factors, Analysis Method

\section{Introduction}

Functional dyspepsia (FD) is a group of common clinical syndrome, mainly for persistent or repeated upper abdominal pain, epigastric burning or discomfort, postprandial fullness and upper abdominal distension, early satiety and so on. FD in the digestive system disease accounts for about $20 \%$ to $40 \%$, outpatient accounted for more than $50 \%$, although the endoscopic examination did not find the structure of the digestive tract abnormalities, and no other symptoms can explain the organic, systemic or metabolic Sexually transmitted diseases. The pathophysiological mechanism is not very clear, may be associated with gastrointestinal motility dysfunction, visceral hypersensitivity, gastric receptivity impairment, gastrointestinal hormone abnormalities, Helicobacter pylori infection and psychosocial disorders and other factors. Among them, the social and psychological factors on the impact of FD patients can not be ignored, mainly including anxiety, depression, somatization and neuroticism, may change the gastrointestinal motility and / or improve the body's visceral sensitivity and 
aggravate the symptoms of indigestion in recent years Hot research at home and abroad.

\section{Subjects}

According to Rome III criteria included in the 207 patients with FD, 69 males and 138 females, aged 25 to 65 years, median age 43 years (37 to 51 years), duration of 6 months to 21 years, the median duration of 24 months (9 to 62 months). There were 48 cases of PDS (early satiety and postprandial fullness discomfort), 53 cases of EPS (upper abdominal pain and epigastric burning sensation), 106 cases of PDS and EPS overlap. All patients were from November 2010 to December 2011 in North Sichuan Medical College Affiliated Hospital of Gastroenterology clinic FD patients were receiving blood, biochemical and other laboratory tests and endoscopy, abdominal hepatobiliary and pancreatic spleen B ultrasound examination To exclude organic disease, and the development of exclusion criteria: (1) less than 18 years of age; (2) endoscopic see a clear organic disease such as peptic ulcer, tumor, bleeding, esophagitis or vascular disease; (3) suffering from mental illness, (5) the recent use of anticholinergics, antispasmodic drugs, aspirin and other non-steroidal anti-inflammatory drugs; (6) nearly two weeks to observe the symptoms of dyspepsia have self-mitigation (5), the incidence of dyspepsia, dyslipidemia, diabetes, (8) can not be independent description of subjective symptoms; (9) within the past six months did not participate in other clinical trials of those who test. Other serious illnesses or serious adverse reactions during the course of medication may affect the course of the test. FD symptoms often associated with IBS (Irritable BowelSyndrome), GERD (Gastro-Esophageal Reflux Disease) and other functional gastrointestinal clinical manifestations overlap, this test allows IBS and GERD symptoms and FD symptoms overlap phenomenon, but the selected FD patients In order to burn or acid-based, after treatment of PPI symptoms of chest pain were relieved were excluded, and functional dyspepsia with abdominal pain, constipation, diarrhea or chest pain were included in the pilot study.

\section{Research Methods}

A total of 207 patients with PDS and PDS overlap EPS patients to be motility drug mosapride and antacid lansoprazole treatment, EPS only lansoprazole treatment (mosapride $5 \mathrm{mg}$ orally, 3 times a day , 30 minutes before meals, lansoprazole $30 \mathrm{mg}$ orally, 2 times a day, 30 minutes before meals), a total of 4 weeks of treatment. NDI and symptoms of dyspepsia before and after treatment were recorded. After 4 weeks of treatment, the patients were evaluated by faceto-face or telephone follow-up, and were divided into two groups: the treatmenteffective group and the control group In the invalid group, the differences of 
somatization symptoms, depression, anxiety and life economic pressure were compared between the two groups before treatment.

Mainly for the clinical symptoms (dyspepsia and somatization symptoms), depression, anxiety and economic pressures, such as the assessment of life. Grade 0: 0 points, asymptomatic; Grade 1: dyspepsia symptom score scale: the main observation of patients with abdominal distension, early satiety, postprandial fullness discomfort, abdominal pain or discomfort and epigastric burning sensation and other symptoms, 2 points, moderate, discomfort affecting daily activities such as work, sleep; 3: 3 points, severe, can not be carried out daily activities such as work, sleep. 1, mild, aware of the symptoms but can tolerate; Headache, chest pain, dizziness, palpitations, shortness of breath, fatigue, insomnia, constipation, or diarrhea (eg, upper abdominal pain, back pain, arm, leg or arthralgia). And other somatization symptoms [11], divided into no effect, a little impact and more impact of three grades, a total of 15 projects, each $0-2$ points, range 0-30 points. In this study, additional observation of patients with dry mouth, globus, sore throat, abdominal pain, poor spirit, numbness, tingling and all other indicators, a total of 21, grading and rating and PHQ-15 the same. Nepean Dyspepsia Index (NDI): A specific quality-of-life scale for the evaluation of dyspepsia symptoms, which includes stress, daily activities, eating / drinking, cognitive / restraint, work / study, Each item is divided into five, the assessment score range 2-10 points, has been widely used in FD-related research.

Body mass index (BMI): to measure the degree of fat and lean body. BMI index $=$ weight $(\mathrm{kg})$ in addition to height $(\mathrm{m})$ of the square $(\mathrm{Kg} / \mathrm{m} 2)$. BMI $=18 \sim$ 25 ; overweight: $\mathrm{BMI}=25 \sim 30$; obesity: $\mathrm{BMI}$ index $>30$, normal weight: $\mathrm{BMI}=$ 18-25; Economic stress: the degree of stress in patients with self-conscious economic life is divided into four levels, one point, larger, large, choose one of them into the scope of the study.

The normal distribution was expressed as the mean \pm standard deviation $(\chi x \pm$ s), and the non-normal distribution was expressed as median or interquartile range (M 25\% -75\%). The rate of comparison was expressed as $\chi 2$ Linear regression and Logistic regression were used to analyze the predictive factors of treatment effectiveness and the possible factors affecting NDI, clinical general information, somatization symptoms and clinical data, and the correlation analysis was made by using the Pearson (normal distribution) or Spearman (nonnormal distribution) Symptom severity, etc. into the regression analysis model (entry method or backward method). The analysis software SPSS13.0 was used. All tests were bilateral, with $\mathrm{P}<0.05$ for the difference was statistically significant.

\section{Results analysis}

The total effective rate was $50.7 \%(105 / 207)$. The effective rate of EPS was $39.6 \%(21 / 53)$.There were no significant difference between the two groups (P> $0.05)$, PDS effective rate was 56.3\% (27/48), PDS and EPS overlap efficiency was $53.8 \%(57 / 106)$. Clinical characteristics of the two groups of patients in 
Table 1. In the effective treatment group, there were 39 cases with no economic stress, 33 cases with a little stress, 29 cases with great pressure and 4 cases with great pressure. In the ineffective treatment group, there were only 6 cases of economic stress, $(\mathrm{P}<0.05)$. The results showed that there were significant differences between the two groups $(\mathrm{P}<0.05)$, and there was significant difference between the two groups $(\mathrm{P}<0.05)$, and there were 44 cases of pressure, 27 cases of large pressure and 25 cases of great pressure. The $\chi 2$ test was 41.015 , The presence and extent of FD may be an important factor influencing the efficacy of FD. There were 53 cases of mild dyspepsia, 104 cases of moderate degree and 50 cases of severe symptoms. The difference between the effective group and the ineffective group was significant $(\chi 2=6.021, \mathrm{P}=0.049, \mathrm{P}<0.05)$. There were no statistically significant differences in age, sex, duration of disease, body mass index and Hp status between the two groups.

The total incidence of depression and anxiety disorder in FD patients was $55.6 \%$ and $60.4 \%$, respectively, which was significantly higher than that of the effective group $(65.7 \%$ VS $45.7 \%, 73.5 \%$ vs $47.6 \%)$. Among them, moderate and severe depression and anxiety disorder were $33.3 \%$ and $35.3 \%$ in the total FD patients, respectively, and the proportion in the ineffective group was significantly higher than that in the effective group (depression: $46.1 \%$ vs $21.0 \%$; anxiety: $50 \%$ vs $21.0 \% \mathrm{X} 2=22.588, \mathrm{P}=0.000$ ), suggesting that depression and anxiety may have an important effect on the efficacy of FD. X2 $=16.262, \mathrm{P}=$ 0.001 ; anxiety disorder;

NDI and its sub-terms and depression, anxiety, PHQ-15 and other correlation analysis, Table 5 shows the invalid group depression, anxiety, PHQ-15, economic life stress, symptom severity and overall NDI score was negatively correlated Depression, anxiety, PHQ-15, economic stress and symptom severity were negatively correlated with stress. Depression, anxiety and symptom severity were negatively correlated with daily activities; depression, anxiety, economic stress, symptom severity and There was a negative correlation between anxiety and work / study, but there was no correlation between age, gender and course of disease and the NDI total score of the ineffective group. There was a negative correlation between anxiety and work /

FD is a common functional gastrointestinal disease, its incidence is higher, the performance of dyspepsia and repeated episodes of multiple symptoms, and accompanied by some somatization symptoms, objective examination revealed no significant organic disease, Patients with psychosomatic distress, seriously affecting the quality of life of patients, but often the lack of effective treatment. In recent years, with the biological-psychological-social medical model in-depth exploration and practice, found that FD patients with psychological and social factors and their symptoms of indigestion are related, and even affect the patient's gastrointestinal function, pain, health care resources, Social status, health-related quality of life, etc. VanOudenhove also found that psychological stress (unemployment), mental disorders, childhood abuse history, social support, coping style and FD may be related to, Patients traveled to hospitals and affected their reporting on the severity of the disease, resulting in a significant waste of medical resources. Although the above-mentioned psychosocial factors on the 
occurrence of FD is so important, but its impact FD mechanism is not yet fully understood, may be expressed as depression, anxiety and other emotional disorders can gastrointestinal motility and its secretory function disorders, Or weaken, resulting in the occurrence of FD, and FD symptoms can reverse the role of the patient to produce depression, anxiety and other adverse mood changes, the two cause each other to form a vicious cycle, leading to FD patients frequent unnecessary medical examination, Long treatment, or even fear of cancer psychology, but also damage their health-related quality of life. Related to the study also reported that a variety of emotional-related psychological disorders can affect the normal activities of the central nervous system, disturbing autonomic nerve function, abnormal regulation of the brain axis, affect the intestinal endocrine system, nervous system and immune system function, reduce gastric emptying and certain Gastrointestinal hormone secretion, resulting in gastrointestinal dysfunction, leading to the occurrence of FD clinical symptoms.

The total effective rate of FD patients was $50.7 \%$. The somatization symptoms such as fatigue, dry mouth, dizziness, sore throat and abdominal pain may affect FD patients. The total incidence of depression and anxiety disorder was $55.6 \%$ $(65.7 \%$ vs $45.7 \%)$, anxiety disorder $(73.5 \%$ vs $47.6 \%)$, and there was significant difference between the two groups (all $\mathrm{P}<0.05$ ), suggesting that the incidence of depression was significantly higher in the treatment group than in the control group Somatization symptoms, depression and anxiety disorders may be an important factor affecting the efficacy of FD.

\section{Conclusion}

Our results showed that: (1) The factors that may affect the efficacy of conventional prokinetic drugs and antacids in the treatment of FD are depression, anxiety, NDI, somatization, economic stress and weight loss, among which NDI and somatization Symptoms are mainly fatigue, dry mouth, dizziness, sore throat, abdominal pain, palpitations and other performance may affect the FD effect is more important factors. Dry mouth, sore throat, headache, abdominal pain, dizziness, poor spirit, numbness, tingling, fatigue may be the impact of PDS effects of PDS may affect the efficacy of PDS sub-group of patients, dry mouth, abdominal pain, dizziness, insomnia, Factors Affecting Efficacy of Overlapping. No factors that may affect the efficacy of EPS were found. (2) FD patients with depression, anxiety disorders and somatization, mixed with multiple factors may lead to FD patients with severe overall symptoms, health-related quality of life damage, affecting the overall efficacy.

\section{References}

[1] Luo Jinbo, Ju Jian. Progress in the pathogenesis of functional dyspepsia. Medical Review, 11(3), pp. 14-17, 2011 
[2] Yang Yingjun, Wen Conghui .Paloxetine hydrochloride in the treatment of functional dyspepsia in 97 cases. China Medical Frontier, 18(6), pp. 16-25, 2009 [3] Li Hua. A randomized, double-blind, placebo-controlled study of mosapride in the treatment of postprandial dysphoria syndrome and epigastralgia syndrome. Journal of Gastroenterology, 15(3), pp. 15-17, 2015

[4] Zhang Cunliang, Li Yanhong.Efficacy of antidepressants in the treatment of functional dyspepsia. Chinese Journal of Misdiagnosis, 23(2), pp. 15-21, 2006

[5] Zhang Xiaoguang, Hu Pinjin, Wang Qiyi, Lin Jinkun. Psychological factors and autonomic nerve function in patients with functional dyspepsia. Gastroenterology, 5(2), p12-22, 2005 